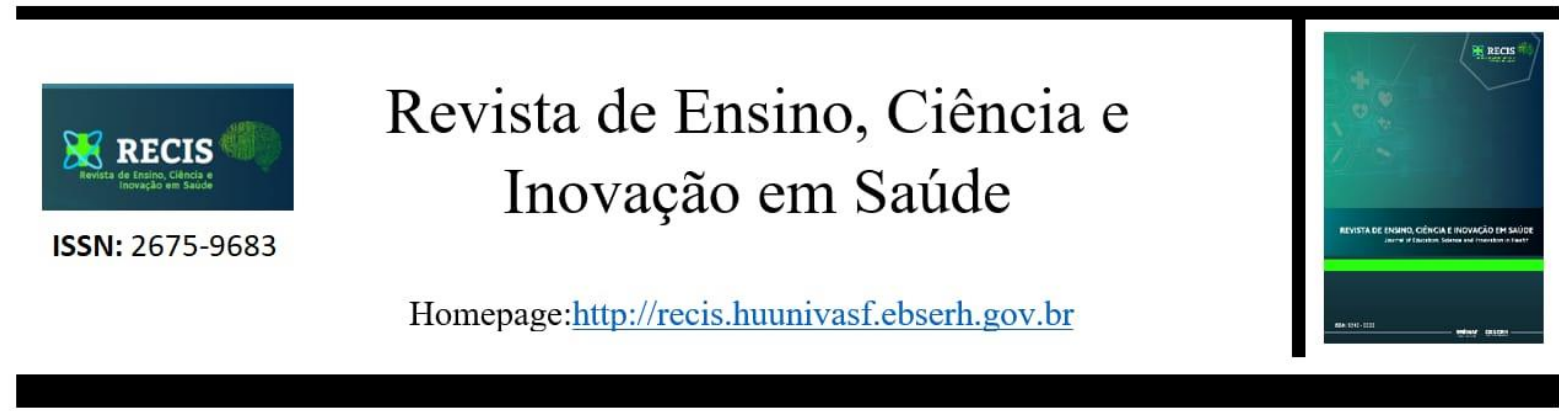

\title{
O papel da Telessaúde na pandemia da Covid-19: um relato de experiência da educação física no contexto hospitalar
}

\section{The role of Telehealth in the Covid-19 pandemic: An experience report of physical education in the hospital contexto}

\author{
Paula Andreatta Maduro', Ammanda Nathally Fernandes Souza², Helder Nunes \\ Lopes $^{3}$, Lucas Felipe Miyasato², Márcia Ribeiro dos Santos², Nathan Leonardo Gomes \\ Costa $^{2}$, Souzeni Silva de Brito ${ }^{2}$, Luiz Alcides Ramires Maduro ${ }^{4}$
}

1Profissional de Educação Física, Hospital Universitário da Universidade Federal do Vale do São

Francisco - HU-UNIVASF, Petrolina-PE, Brasil. ${ }^{2}$ Discente, Colegiado de Educação Física,

Universidade Federal do Vale do São Francisco - UNIVASF, Petrolina-PE, Brasil. ${ }^{3}$ Analista de Tecnologia da Informação, Hospital Universitário da Universidade Federal do Vale do São Francisco - UNIVASF/EBSERH, Petrolina-PE, Brasil. ${ }^{4}$ Docente, Colegiado de Educação Física, Universidade Federal do Vale do São Francisco - UNIVASF, Petrolina-PE, Brasil.

Autor para correspondência: paula.maduro@ebserh.gov.br

Artigo recebido em 28/09/2021e aceito em 24/11/2021

\section{RESUMO}

A Telessaúde se caracteriza como a oferta de serviços de atenção à saúde, em situações geográficas críticas ou em caso de situações excepcionais, como foi o caso da Covid-19. Desta forma foi utilizada a atividade física de maneira remota como estratégia de enfrentamento ao sedentarismo dos idosos, participantes do Projeto Saúde do Idoso do HU-Univasf, que tiveram que interromper as atividades desde o início da pandemia. Neste contexto, o objetivo deste relato de experiência é descrever a implantação da Telessaúde, no contexto hospitalar, como estratégia para ministrar aulas de atividades físicas aos idosos, durante o período da Covid-19. Participaram deste novo formato de aula remota 20 idosos, a profissional de educação física do HU-Univasf e cinco estudantes do colegiado de Educação Física da Universidade Federal do Vale do São Francisco (CEFIS-Univasf). O formato das aulas teve boa aceitação, apesar da barreira tecnológica existente na execução do projeto, visto que se verificou a aderência de $90 \%$ dos participantes selecionados, desde a implementação do modelo remoto. Foi constatado através de relatos, a melhora da capacidade física, da cognição e dos fatores psicológicos dos idosos. Bem como, a experiência aprimorou o processo pedagógico virtual da equipe de trabalho envolvida, se caracterizando como uma real possibilidade de adesão à prática de atividade física e proporcionando a melhora da qualidade de vida da população idosa.

Palavras-chave: Telessaúde; e-Saúde; Atividade Física; Idoso; Covid-19.

\section{ABSTRACT}

Telehealth is characterized as the provision of health care services in critical world situations or in exceptional situations, as the case with Covid-19. Thus, physical activity was used remotely as a strategy to face the sedentary lifestyle of the elderly people, participants of the Elderly Health Project of the HU-Univasf, who had to interrupt their activities since the beginning of the Covid-19 pandemic. In this context, the objective of this experience 
report is to describe the implementation of Telehealth in the hospital context as a strategy to teach physical activity classes to elderly people during the period of Covid-19. Twenty seniors, the physical education professional from the HU-Univasf and five students from the Physical Education collegiate of the Federal University of Vale do São Francisco (CEFIS-Univasf) participated in this new remote class format. The format of the classes was well accepted despite the technological barrier existing in the execution of the project, since $90 \%$ of selected participants adhered to since the implementation of the remote model. It was found through reports, the improvement of physical capacity, cognition and psychological factors of the elderly people. As well a, the experience improved the virtual pedagogical process of the work team involved, characterizing itself as a real possibility of adherence to the practice of physical activity and providing an improvement in the quality of life of the elderly population.

Keywords: Telehealth; e-Health; Physical Activity; Elderly; Covid-19.

\section{INTRODUÇÃO}

O Hospital Universitário da Universidade Federal do Vale do São Francisco (HU-UNIVASF) localiza-se na cidade de Petrolina, no sertão pernambucano. É administrado pela Empresa Brasileira de Serviços Hospitalares (EBSERH) desde 01 de fevereiro de 2015. Considerado unidade de referência para os municípios da Rede Interestadual de Atenção à Saúde do Médio do São Francisco, com atenção à urgência e emergência, que incluem politraumatismo, neurologia e neurocirurgia, traumato-ortopedia, cirurgia geral, cirurgia vascular, cirurgia bucomaxilofacial, clínica médica e cirurgia plástica restauradora, com atendimento multidisciplinar das equipes de saúde. Além disso, é referência de ensino para os cursos da saúde oriundos da própria Univasf e de outras instituições de ensino superior da região.

Neste contexto, a Educação Física está inserida no setor de reabilitação desta instituição e desenvolve diversas atividades, dentre elas, o Projeto de Saúde do Idoso, com pacientes que são egressos deste hospital ou recrutados por demanda espontânea na comunidade. Tal projeto tem como objetivo oportunizar a melhora da qualidade de vida dos idosos participantes por meio de atividades físicas, desenvolvendo exercícios de força, equilíbrio, flexibilidade e cognição. As atividades práticas tiveram início em março de 2018 e eram realizadas dois dias por semana, atendendo cerca de trinta (30) idosos, com faixa etária entre 60 a 80 anos, saudáveis ou com presença de hipertensão arterial sistêmica, diabetes, obesidade, câncer e dislipidemia.

Em dezembro de 2019, o primeiro caso de coronavírus foi detectado em Wuhan, capital da província chinesa de Hubei. Desde então o vírus contaminou e matou milhões de pessoas pelo mundo ${ }^{1}$. No Brasil, foram cerca de 22 milhões de pessoas infectadas e mais de $613 \mathrm{mil}$ mortes. Em fevereiro de 2020, o Ministério da Saúde confirmou o primeiro caso de COVID19 no Brasil, um idoso de 61 anos de idade que esteve em viagem na Itália.

Em 06 de fevereiro de 2020, o Congresso Nacional decretou a Lei $\mathrm{n}^{\circ} 13.979^{1}$, que dispõe sobre as medidas a serem adotadas para o enfrentamento à Covid-19.

Em 11 de março de 2020, a Organização Mundial da Saúde (OMS) classificou a Covid-19, como doença respiratória causada pelo vírus SARS-CoV-2, como uma pandemia ${ }^{2}$. O SARS-CoV-2, por ser transmitido principalmente pelo ar, é um agente com uma alta taxa de contágio, seus sintomas podem ser extremamente variados ${ }^{3}$ e graves, até mesmo letais para pessoas dos grupos de risco, como por exemplo pessoas com comorbidades, com baixa imunidade e população idosa ${ }^{4}$, fazendo necessário que as atividades presenciais fossem interrompidas, como medida preventiva. Outras medidas preventivas são o uso correto de máscaras faciais, o isolamento social, a higienização constante das mãos e superfícies.

Em 14 de março de 2020, o governador do estado de Pernambuco (onde se localiza o Projeto de Saúde do Idoso) sancionou o Decreto $\mathrm{n}^{\circ} .48 .809^{5}$ que dispõe sobre as medidas a serem adotadas para contenção da pandemia, corroborando com a Lei $n^{\circ}$ 13.979. Em 18 de março de 2020, o Governador sancionou outro Decreto de $\mathrm{n}^{\circ} 48.822^{6}$, dando início ao Lockdown (confinamento), bem como a suspensão de atividades de academias de ginástica e similares. Sendo assim, todas as atividades, incluindo o Projeto de Saúde dos Idosos foram interrompidas para preservar os idosos da Covid-19. Vista disso, a classificação do nível de atividade física desses participantes passou de ativos a sedentários. E, impulsionados pela falta de atividade em 
função do isolamento, as questões físicas e funcionais, assim como a saúde mental. Essas ações ocasionaram consequências no bem-estar destes idosos, potencializadas principalmente durante o afastamento social ${ }^{7}$ e elevaram os riscos relacionados às doenças crônicas e a Covid para o grupo de idosos com comorbidades $^{8}$.

\section{OBJETIVO}

Este relato de experiência tem como objetivo descrever a implantação da Telessaúde no contexto hospitalar como estratégia para ministrar aulas de atividades físicas aos idosos participantes do Projeto Saúde do Idoso do HUUnivasf, durante o período da pandemia da Covid-19.

\section{RELATO DE EXPERIÊNCIA}

Como estratégia para atender as demandas na área da saúde como consulta, assistência e direcionamentos médicos utilizando a internet e tecnologias similares, a e-Saúde (E-Health) foi criada como mecanismo para acelerar a dinâmica entre profissionais da saúde e pacientes. Com a oficialização e crescente consolidação desde os anos 2000, a eSaúde é um método aprovado por médicos e pacientes, se tornando comum principalmente nos países desenvolvidos do continente europeu graças às políticas governamentais de incentivo ao uso de tecnologia na área da saúde $^{9,10,11}$.

A aplicação da e-Saúde, como: Telessaúde, Teleterapia, Teleconsulta, Telemedicina entre outros campos, ficaram mais comuns com o passar dos anos ${ }^{12}$, sendo mais utilizadas durante a pandemia. No Brasil, a e-Saúde é estimulada pelo Ministério da Saúde (MS) conectando serviços oferecidos pelo Sistema Único de Saúde (SUS) ${ }^{13}$. Programas estendem seus serviços para suportar atividades de treinamento $\mathrm{e}$ de informação em saúde para profissionais multidisciplinares e pacientes no ambiente digital ${ }^{14,15}$.

A Telessaúde emerge como alternativa utilizada para o retorno das atividades físicas dos idosos mantendo o distanciamento social necessário para esta população. Este método utiliza recursos tecnológicos para aproximar quem necessita de assistência à saúde com quem pode ofertá-la ${ }^{16}$. Além disso, a Telessaúde tem como benefícios a facilidade de uso, a possibilidade de comunicação com pessoas distantes a um baixo custo financeiro ou com restrição de mobilidade, disponibilidade de serviço em áreas rurais e excelente aceitação por pacientes ${ }^{17,18}$.

Em 2020, a pandemia da Covid-19 interrompeu as atividades presenciais, acelerando a necessidade da e-Saúde. Logo, em tempos pandêmicos é de consenso global o aproveitamento da Telessaúde como meio de acesso a regiões de recursos limitados ao combate da Covid-19. Com objetivo de viabilizar serviços como triagem, diagnósticos, tratamentos, acompanhamento médico e reabilitação, a Telessaúde ocupa uma lacuna levantada pelo distanciamento social, mantendo os serviços de saúde funcionantes e mais seguros ${ }^{19,20,21}$. Apoiados pela Associação Brasileira de Telemedicina e Telessaúde $(\mathrm{ABTms})^{22}$, profissionais de Educação Física, relataram em Webnars ABTms a utilização da Telessaúde em projetos voltados à saúde da comunidade e os benefícios da mesma, discutindo como podem melhorar essa nova forma de trabalho, diante das experiências dos profissionais e alunos que fazem uso da ferramenta (ABTms, 2021).

Relatos da literatura ${ }^{20,23,24}$ descrevem o uso da Telessaúde como componente fundamental na resposta às dificuldades impostas durante a pandemia da Covid-19. A necessidade de continuar ofertando saúde foi o fator responsável por acelerar a implementação desta ferramenta para o retorno do projeto de atividade física dos idosos. Com o avanço da tecnologia e o uso de smartphones com acesso à internet por boa parte dos idosos, o contato para realização das atividades remotas foi planejado levando as possibilidades dos mesmos no novo ambiente.

Em função destas colocações, a área da Educação Física, do setor de reabilitação do HU-Univasf, vem utilizando a Telessaúde como alternativa de retorno do exercício físico regular dos idosos do projeto. São utilizadas atividades remotas que tem como objetivo resgatar as perdas físicas e mentais decorrentes do isolamento e da falta de exercícios físicos, levando em consideração as dificuldades do ambiente virtual e do tempo sem participarem de atividades do projeto. Assim, o objetivo do retorno das atividades nesse novo formato foi 
evitar o ganho de peso corporal, a perda de massa magra e quedas, promover o ganho de força, bem como, desacelerar a perda da função cognitiva.

No projeto presencial, interrompido em março de 2020, os idosos realizaram avaliação antropométrica (massa corporal, estatura, Índice de massa corpórea), teste de caminhada de 6 minutos $^{25}$, Questionário do Estado Geral de Saúde e Uso de Medicamentos e para avaliar a qualidade de vida relacionada à saúde foi utilizado o Short Form Health Survey $36^{26}$ (SF36), instrumento validado e atualizado no Brasil $^{27,28}$. O SF-36, é composto por duas dimensões: física e mental, sendo organizado nas escalas de capacidade funcional (10 itens), aspectos físicos (4 itens), dor (2 itens), estado geral de saúde (5 itens), vitalidade (4 itens), aspectos sociais ( 2 itens), aspectos emocionais (3 itens) e saúde mental (5 itens). Os objetivos das práticas corporais foram a melhora da qualidade de vida, por meio de tarefas que desenvolveram força, equilíbrio, coordenação, flexibilidade e cognição, além da saúde mental, promovida por meio da socialização.

No modelo remoto foram mantidos os objetivos e realizadas as seguintes avaliações: Questionário de Estado Geral de Saúde e Uso de Medicamentos e o SF-36, que foram aplicados antes do início das atividades remotas e três meses após a realização das mesmas. Estas atividades são ministradas via plataforma Google Meet, com o link disponibilizado diariamente nos grupos de Whatsapp, que foram criados para facilitar a comunicação mútua entre os idosos, a professora e os estagiários de educação física. Os ministrantes das aulas se reúnem em uma sala da Policlínica do HU-Univasf equipada com um webcam conectado a um computador com acesso a internet. Com esses equipamentos mencionados, a equipe foi capaz de ministrar aulas adequadas para os idosos. O material utilizado pelos idosos, em sua maioria, são alternativos e de uso doméstico, tais como: cadeiras, garrafas pets cheias de areia ou água, colchonetes, bolas de plástico, toalha $\mathrm{e}$ almofada. Durante a aula, a equipe realiza orientações sobre a execução dos exercícios, bem como a realização dos movimentos de forma segura executados em ambientes abertos, afastados de mesas e quinas, evitando pisos molhados e escorregadios; quanto a vestimenta: calçando tênis e trajando roupas apropriadas a mobilidade. Quanto a possíveis relatos de dor antes, durante ou após o exercício, existe um canal de comunicação entre os idosos e a equipe para minimizar este fato. Algumas observações são realizadas pela equipe, tais como: em caso de exercícios que utilizem uma cadeira, certificar-se de que a mesma se encontra bem apoiada na parede e que não tenha rodas; em caso de exercícios no solo, utilizar uma toalha grande, evitando colchões altos, o que irá proporcionar uma prática correta.

\section{REFLEXÃO SOBRE A EXPERIÊNCIA}

A iniciativa das aulas de atividade física em formato remoto para os idosos do projeto apresentou algumas barreiras, as quais foram desafiadoras, tanto para os idosos quanto para a profissional e os estudantes. De maneira evidente, a maior de todas foi a falta de conhecimento tecnológico dos idosos participantes do projeto. Tal característica fez com que alguns idosos não conseguissem entrar no ambiente virtual para ter acesso às aulas. Ademais, controles básicos como ligar/desligar o microfone e câmera, aumentar/diminuir o volume do dispositivo se tornaram tarefas difíceis para eles. Estas dificuldades no início das aulas remotas comprometeram a qualidade e/ou andamento das sessões das mesmas, entretanto, com o passar do tempo, os idosos aprenderam a lidar com essas barreiras e hoje, o andamento e a qualidade das aulas estão adaptados para o momento.

Partindo para outra dificuldade existente, temos a impossibilidade da interação presencial entre a equipe que ministra as atividades e os alunos. Em algumas ocasiões, os idosos sentem dificuldade de realizar um determinado movimento, para tal, como método de correção utilizamos o recurso dos comandos verbais e visuais, o que proporcionou a correção dos principais erros na realização dos exercícios e postura. Ainda pensando nas dificuldades, é nítido que o processo de socialização foi afetado, em função da necessidade do isolamento social para esta população de risco ${ }^{29}$. Os idosos no projeto em sua versão presencial tinham relações de amizade $e$ esse vínculo proporcionou confraternizações sociais, momentos esses que favoreciam a aproximação dos mesmos. De maneira positiva, quando a Telessaúde foi adotada a socialização de forma presencial foi 
transferida para a forma remota, possibilitando a troca de afeto e preocupação entre os alunos, além das conversas pelo grupo do whatsapp, antes e ao final das aulas remotas.

A Telessaúde foi uma estratégia operacional em tempos pandêmicos para a volta da atividade física dos idosos, que eram ativos e tornaram-se sedentários no período de março de 2020 a março de 2021, quando retornaram à atividade de forma remota. Mesmo com algumas adversidades, os participantes relataram mais pontos positivos que negativos. A melhora na qualidade de vida, da capacidade funcional e da saúde mental ficou evidente nos depoimentos dos mesmos. Os idosos relataram redução de dor; melhor desempenho em atividades básicas e instrumentais de vida diária; melhora na flexibilidade, na cognição, redução dos sintomas de ansiedade e depressão. Em outra percepção, esses relatos reforçam a ideia de que envelhecer, na verdade, se trata de um período de constante progresso na capacidade de aprendizado e adaptação ao mundo moderno, isto mostra ser possível a independência do idoso no mundo tecnológico ${ }^{30}$.

Quanto ao condicionamento físico e a cognição, os idosos citaram que no início das aulas remotas alguns não conseguiam realizar os exercícios por fadiga muscular e/ou déficit cognitivo. Para a melhora dos resultados físicos, da capacidade funcional, fatores psicológicos e cognitivos foi construído o plano de aula com as atividades conforme as necessidades do grupo, controlando o volume e a intensidade dos exercícios, o que possibilita uma melhor participação e correta execução dos movimentos evitando a fadiga muscular.

\section{CONCLUSÃo}

Os objetivos do modelo da Telessaúde desenvolveram habilidades para realizar as atividades de vida diária, o que possibilitou, neste período pandêmico uma boa aceitação por parte dos idosos, sugerindo que a implementação da mesma foi bem-sucedida, com aderência de 20 participantes em maio de 2021. Durante os seis meses do projeto houve um abandono de $10 \%$ dos participantes nas atividades, o que indica uma alta adesão ao projeto, inferindo como um ponto positivo evidenciado. Para a Educação Física, a inserção na Telessaúde foi uma janela aberta para novas oportunidades, devendo ser explorada por profissionais, estudantes e pesquisadores da área, objetivando atingir e melhorar a realidade dos alunos.

Pode-se concluir que as atividades remotas tiveram boa aceitação na rotina dos idosos conforme feedback dos mesmos, assim como, na melhora na qualidade de vida em parâmetros físicos e mentais. A oportunidade de participar das práticas, após tantas dificuldades impostas pela pandemia do Covid$19 \mathrm{fez}$ com que as aulas fossem celebradas pelos participantes. A nova perspectiva da Telessaúde foi tão bem aceita que em alguns casos é o modelo escolhido pelos idosos quando indagados sobre o retorno das aulas presenciais.

Para isto, faz-se necessário pensar em plataformas e aplicativos específicos para que os atendimentos, aulas e consultorias possam ter melhor qualidade e trocas para os pacientes e alunos. Ademais, estudos apontam que para a geração e manutenção de uma educação digital é necessária uma autoeficácia desses participantes na alfabetização em saúde eletrônica (eHL) ${ }^{31,32}$, visto que as tecnologias estão em constante atualização. Logo, como estratégias para a eHL, a troca de informações através de redes sociais ou comunidades online podem fortalecer o vínculo de idosos com as ferramentas.

Essa aproximação do público idoso com o meio digital possibilitou maiores correções entre quem ministra as aulas online e os idosos, podendo haver redução de erros na execução dos exercícios ${ }^{32,33,34}$.

Em relação aos estudantes que participaram do projeto, foi um período enriquecedor para formação profissional dos mesmos, pois a Telessaúde continuará a existir mesmo após o fim da pandemia da Covid-19. Como a Educação Física é uma área que está em constante atualização, novas estratégias são sempre bem-vindas, logo acreditamos que os profissionais que não tiveram contato com a Telessaúde estão a "alguns passos" atrás dos que utilizaram esta ferramenta com eficiência e estratégia de aproximação com o público.

O HU-Univasf ao promover o Projeto do Idoso aproxima as instituições da comunidade, preservando e expandindo o atendimento aos pacientes. Além disso, cria-se uma nova oportunidade de adesão a exercícios físicos, desviando-os da zona do sedentarismo, da oportunidade de intervenção não 
farmacológica e de situações hospitalares críticas, a qual proporciona uma melhor condição de saúde para os idosos.

\section{REFERÊNCIAS}

1 - Brasil. Congresso Nacional. Lei $\mathrm{n}^{\mathrm{o}}$ 13.979, de 6 de fevereiro de 2020. Dispõe sobre as medidas para enfrentamento da emergência de saúde pública de importância internacional decorrente do coronavírus responsável pelo surto de 2019. Brasília: Planalto; 2020.

2 - Organização Mundial da Saúde. Rolling updates on coronavirus disease (COVID-19). World Health Organization [Internet]. 2020. [citado 2021 ago 26]. Disponível em: https://www.who.int/emergencies/diseases/ novel-coronavirus-2019/events-as-theyhappen.

3 - Iser BPM, Silva I, Raymundo VT, Poleto MB, Schuelter-Trevisol F, Bobinski F. Definição de caso suspeito da COVID-19: uma revisão narrativa dos sinais e sintomas mais frequentes entre os casos confirmados. Epidemiol. Serv. Saúde. 2020;29(3);1-11. https://doi.org/10.5123/S1679-

49742020000300018

4 - Ministério da Saúde. Secretaria de Vigilância em Saúde. Centro de Operações de Emergências em Saúde Pública. Especial: doença pelo coronavírus 2019. Bol Epidemiol 7 [Internet]. 2020 [citado 2020 jun 1]. Disponível em: $\quad$ http://www.cofen.gov.br/wpcontent/uploads/2020/04/Boletim-07-MS-0604-2020.pdf.pdf

5 - Brasil. Governo do Estado de Pernambuco. Decreto $\mathrm{n}^{\circ}$. 48.809, de 14 de março de 2020. Regulamenta, no Estado de Pernambuco, medidas temporárias para enfrentamento da emergência de saúde pública de importância internacional decorrente do coronavírus, conforme previsto na Lei Federal $n^{\circ} 13.979$, de 6 de fevereiro de 2020. Recife: Assembleia Legislativa do Estado de Pernambuco; 2020.

6 - Brasil. Governo do Estado de Pernambuco. Decreto $n^{\circ}$. $\mathrm{N}^{\mathrm{o}} 48.822$, de 17 de março de 2020. Altera o Decreto ${ }^{\circ} 48.809$, de 14 de março de 2020, que regulamenta, no Estado de Pernambuco, medidas temporárias para enfrentamento da emergência de saúde pública de importância internacional decorrente do coronavírus responsável pelo surto de 2019, conforme previsto na Lei Federal n ${ }^{\circ} 13.979$, de
6 de fevereiro de 2020. Recife: Assembleia Legislativa do Estado de Pernambuco; 2020.

7- Lima RC. Distanciamento e isolamento sociais pela Covid-19 no Brasil: impactos na saúde mental. Physis. 2020;30(2). https://doi.org/10.1590/S0103-

73312020300214

8 - Dourado SP da C. A pandemia de COVID19 e a conversão de idosos em "grupo de risco". Cadernos de Campo (São Paulo - 1991). 2020;29(supl):153-62.

https://doi.org/10.11606/issn.2316-

9133.v29isuplp153-162

9 - Polinski JM, Barker T, Gagliano N, Sussman A, Brennan TA, Shrank WH. Patients' satisfaction with and preference for telehealth visits. J Gen Intern Med. 2016;31(3):269-75. https://doi.org/10.1007/s11606-015-3489-x 10 - Isautier JM, Copp T, Ayre J, Cvejic E, Meyerowitz-Katz G, Batcup C, Bonner C, Dodd R, Nickel B, Pickles K, Cornell S. Lessons from the COVID-19 pandemic: People's experiences and satisfaction with telehealth during the COVID-19 pandemic in Australia. medRxiv. 2020;09(11):1-21. https://doi.org/10.1101/2020.09.10.20192336 11 - Santos AF... [et al.] Organizadores. Telessaúde: um instrumento de suporte assistencial e educação permanente. Belo Horizonte: Editora UFMG; 2006.

12 - Shaw T, McGregor D, Brunner M, Keep M, Janssen A, Barnet S. What is eHealth (6)? Development of a conceptual model for eHealth: qualitative study with key informants. J Med Internet Res. 2017;19(10):e324. https://doi.org/10.2196/jmir.8106

13 - Ministério da Saúde. O que é Saúde Digital? [Internet]. Brasil [citado 2021 set 02]; Disponível em: https://www.gov.br/saude/ptbr/assuntos/saude-digital

14- Bashshur R, Shannon G, Krupinski E, Grigsby J. The taxonomy of telemedicine. Telemedicine and e-Health. 2011;17(6):48494. https://doi.org/10.1089/tmj.2011.0103

15 - Ministério da Saúde. Saúde Digital e Telessaúde. [Internet]. Brasil [citado 2021 ago 26]; Disponível em: https://www.gov.br/saude/pt-

br/assuntos/saude-digital/telessaude/telessaude 16 - Cottrell MA, Russell TG. Telehealth for musculoskeletal physiotherapy. Musculoskelet. Sci. Pract. 2020; 48:102-193. https://doi.org/10.1016/j.msksp.2020.102193 
17 - Kruse CS, Krowski N, Rodriguez B, Tran L, Vela J, Brooks M. Telehealth and patient satisfaction: a systematic review and narrative analysis. BMJ open. 2017; 7(8): e016242. http://dx.doi.org/10.1136/bmjopen-2017016242

18 - Burkow TM, Vognild LK, Johnsen E, Bratvold A, Risberg MJ. Promoting exercise training and physical activity in daily life: a feasibility study of a virtual group intervention for behaviour change in COPD. BMC Med. Inform. Decis. Mak. 2018;18(1):1-3. https://doi.org/10.1186/s12911-018-0721-8.

19 - Doraiswamy S, Abraham A, Mamtani R, Cheema S. Use of telehealth during the COVID-19 Pandemic: Scoping Review. J Med Internet Res. 2020;22(12):e24087. https://doi.org/10.2196/24087

20 - Campos BH, Alfieri DF, Bueno EB, Kerbauy G, Dellaroza MS, de Araujo Ferreira NM. Telessaúde e telemedicina: uma ação de extensão durante a pandemia. Revista Aproximação. 2020. [citado 2020 Jul 31]; 2(04):24-8. Disponível em: https://revistas.unicentro.br/index.php/aproxim acao/article/view/6578/4499

21 - Caetano R, Silva AB, Guedes AC, Paiva CC, Ribeiro GD, Santos DL, Silva RM. Desafios e oportunidades para telessaúde em tempos da pandemia pela COVID-19: uma reflexão sobre os espaços e iniciativas no contexto brasileiro. Cad. Saúde Pública. 2020;36(5):e00088920.

https://doi.org/10.1590/0102-311X00088920 22 - Associação Brasileira de Telemedicina e Telessaúde [internet] Webinar com Educação Física aponta desafios da categoria regulamentada para atuação na Saúde em 2020. 2021. [citado 2021 ago 26]. Disponível em: https://www.abtms.org.br/pt/webinar-comprofissionais-de-educacao-fisica-apontadesafios-de-uma-categoria-profissionalregulamentada-recentemente-para-atuacao-nasaude/

23 - Hamza Shuja K, Aqeel M, Jaffar A, Ahmed A. COVID-19 pandemic and impending global mental health implications. Psychiatr. Danub. 2020;32(1):32-5.

https://doi.org/10.24869/psyd.2020.32 24 - Silva RS, Schmtiz CA, Harzheim E, Molina-Bastos CG, Oliveira EB, Roman R, Umpierre RN, Gonçalves MR. O Papel da Telessaúde na Pandemia Covid-19: Uma Experiência Brasileira. Ciênc. Saúde Coletiva.
2021;26(6):2149-57.

https://doi.org/10.1590/1413-

81232021266.39662020

25 - Britto RR, de Sousa LA. Teste de caminhada de seis minutos uma normatização brasileira. Fisioterapia em movimento. 2017. [citado 2017 Aug 31]; 19(4). Disponível em: https://periodicos.pucpr.br/index.php/fisio/arti cle/download/18789/18171

26 - Ware JE Jr, Sherbourne CD. The MOS 36item short-form health survey (SF-36). I. Conceptual framework and item selection. Med Care. 1992;30(6):473-483. http://dx.doi.org/10.1097/00005650199206000-00002

27 - Soárez PC, Kowalski CCG, Ferraz MB, Ciconelli RM. Tradução para português brasileiro e validação de um questionário de avaliação de produtividade. Rev. Panam. Salud Publica. 2007;22(1):21-8. https://doi.org/10.1590/s102049892007000600003

28 - Laguardia J, Campos MR, Travassos C, Najar AL, Anjos LA, Vasconcellos MM. Dados normativos brasileiros do questionário Short Form-36 versão 2. Ver. Bras. Epidemiol. 2013; 16(04):889-97. https://doi.org/10.1590/S1415790X2013000400009

29 - Lima Bezerra PC, de Lima LC, Dantas SC. Pandemia da covid-19 e idosos como população de risco: aspectos para educação em saúde. Cogitare Enferm. 2020;25. http://dx.doi.org/10.5380/ce.v25i0.73307 30 - Kachar V. Terceira idade e informática: aprender revelando potencialidades. São Paulo. Editora Cortez. 2003.

31 - Parkinson J, David P, Rundle-Thiele S. Self-efficacy or perceived behavioural control: Which influences consumers' physical activity and healthful eating behaviour maintenance?. J Consumer Behav. 2017;16(5): 413-23.

https://doi.org/10.1002/cb.1641

32 - Rabenbauer LM, Mevenkamp N. Factors in the Effectiveness of e-Health Interventions for Chronic Back Pain: How Self-Efficacy Mediates e-Health Literacy and Healthy Habits. Telemed J E Health. 2021;27(2):184-92. https://doi.org/10.1089/tmj.2019.0301

33 - Norman CD, Skinner HA. eHealth literacy: essential skills for consumer health in a networked world. J Med Internet Res. 2006;8(2):e9.

https://doi.org/10.2196/jmir.8.2.e9 
Revista de Ensino, Ciência e Inovação em Saúde v.2, n.3 (2021) 40-47

ISSN: 2675-9683/DOI: $10.51909 /$ recis.v2i3.178

34 - Tang Y, Tseng HW. Distance learners' self-efficacy and information literacy skills. J.
Acad. Librariansh. 2013;39(6):517-21.

https://doi.org/10.1016/j.acalib.2013.08.008 